\title{
Prediction of Functional Outcome in Post-Hospital Brain Injury Rehabilitation
}

\author{
Frank D. Lewis ${ }^{1}$, Gordon J. Horn ${ }^{2}$ \\ ${ }^{1}$ NeuroRestorative Research Institute, Medical College of Georgia at Augusta University, Augusta, GA, USA \\ ${ }^{2}$ NeuroRestorative Research Institute, College of Medicine, Florida State University, Tallahassee, FL, USA \\ Email: frank.lewis@neurorestorative.com
}

How to cite this paper: Lewis, F.D. and Horn, G.J. (2019) Prediction of Functional Outcome in Post-Hospital Brain Injury Rehabilitation. Journal of Behavioral and Brain Science, 9, 406-416.

https://doi.org/10.4236/jbbs.2019.912031

Received: December 5, 2019

Accepted: December 20, 2019

Published: December 23, 2019

Copyright $\odot 2019$ by author(s) and Scientific Research Publishing Inc. This work is licensed under the Creative Commons Attribution International License (CC BY 4.0).

http://creativecommons.org/licenses/by/4.0/

\begin{abstract}
Post-hospital brain injury rehabilitation programs are afforded limited time to reduce chronic disability resulting from acquired brain injury. Therefore, the purpose of this study was to identify deficit areas resulting from acquired brain injury that have the greatest impact on functional outcomes to enable greater efficiency in rehabilitation programming. Study participants were 1717 persons with acquired brain injury treated in residential post-hospital rehabilitation programs. Participants were assessed at admission and discharge on the MPAI-4. Functional status at discharge was evaluated based on T-scores derived from MPAI-4 discharge participation index items: initiation, self-care and residence. The database of 1717 was randomly divided into two subsets. Items from admission Abilities Index and select person variables were entered into stepwise multiple regression on subset one and then in a hierarchical multiple regression on subset two. Rash analysis demonstrated satisfactory construct validity and internal consistency of admission and discharge MPAI-4 evaluations (Person reliability $\geq 0.90$, Item reliability $=0.99$ ). Both regression analyses revealed that Mobility and Novel Problem Solving accounted for $40 \%$ of the variance in functional outcome, $\mathrm{p}<0.001$. Acquired brain injury results in a myriad of cognitive and physical impairments. Of the many possible deficits, the greatest gains in overall functional outcomes may be achieved by allocating additional treatments aimed at reducing disability in mobility and novel problem solving.
\end{abstract}

\section{Keywords}

Brain Injury, Mayo Portland Adaptability Index Version-4, Rasch Analysis, Outcome, Post-Hospital Brain Injury Rehabilitation

\section{Introduction}

Post-hospital brain injury rehabilitation programs exist to reduce disability and 
improve the overall health status of persons who have experienced an acquired brain injury. A brain injury, whether traumatic or non-traumatic, disrupts normal brain functions resulting in enduring impairment to cognition, communication, mobility and emotional well-being [1]. A brain injury is a chronic condition with an indefinite period of time required to regain or improve function [2]. Current research suggests that with comprehensive multidisciplinary rehabilitation, survivors can realize significant reduction in disability well over a year after injury [3] [4] [5] [6]. However, the majority of specialized brain injury rehabilitation services is accessed from a hospital system and concludes within approximately 2 - 3 months following injury [7]. The need for continued rehabilitative care has resulted in post-hospital services being developed with the purpose of continued restoration of independence over a gradual period of time providing appropriate medical, behavioral, and social supports to sustain and enhance quality of life [7].

Unfortunately, funding for this level of treatment is limited, with only a minority of those in need having access to this care [1]. With limited financial resources allocated for post-hospital brain rehabilitation, it is critical that these programs utilize evidenced based best practice in order to maximize efficiency and effectiveness in achieving positive outcomes. Best practices that shorten the length of treatment time while obtaining meaningful reduction in disability can extend existing resources which may be utilized for others in need.

One approach to developing such best practices is to identify those impairment areas that have the greatest impact on outcome. This would enable a more focused approach to rehabilitative care in which those deficits having the greatest impact on functional independence would be apportioned more frequent treatments of longer duration. Therefore, the purpose of this study was to identify functional deficits that have the greatest impact on outcome following acquired brain injury.

\section{Methods}

\subsection{Subjects}

Participants for the study were selected from a database consisting of 3511 neurologically impaired persons with consecutive discharges from 32 residential and nonresidential post-hospital rehabilitation programs in 24 states from 2011-2018. All programs were part of a network of care for brain injured individuals. These programs included active neurorehabilitation (residential physical and cognitive rehabilitation), neurobehavioral intensive (residential rehabilitation emphasizing reduction of physical and verbal aggression), day treatment (non-residential physical and cognitive rehabilitation) and supported living (long-term residential care emphasizing quality of life). For a detailed description of each of these programs see Lewis and Horn, 2015 [5]. From this inclusive database, 1717 met study inclusion criteria: age $\geq 18$, diagnosis of acquired brain injury and treated in residential neurorehabilitation programs with a minimum 
length of stay $\geq 30$ days. Exclusion criteria included persons receiving treatment for disorders not considered an acquired brain injury (e.g. spinal cord disorder, psychiatric disorder).

The demographic characteristics of the study sample are presented in Table 1.

Table 1. Demographics for study participants.

\begin{tabular}{|c|c|}
\hline \multicolumn{2}{|l|}{ Gender } \\
\hline Male & $76 \%$ \\
\hline Female & $24 \%$ \\
\hline \multicolumn{2}{|l|}{ Age (years) } \\
\hline Mean & 49.9 \\
\hline $\mathrm{SD}$ & 15.0 \\
\hline Range & $18-89$ \\
\hline \multicolumn{2}{|l|}{ Type of Injury } \\
\hline Traumatic Brain Injury & $73 \%$ \\
\hline Cerebral Vascular Accident & $16 \%$ \\
\hline Anoxic/hypoxic & $5 \%$ \\
\hline Encephalopathy & $4 \%$ \\
\hline Tumor & $2 \%$ \\
\hline \multicolumn{2}{|l|}{ Time Since Injury (months) } \\
\hline Mean & 22.9 \\
\hline $\mathrm{SD}$ & 59.8 \\
\hline Range & $1-564$ \\
\hline \multicolumn{2}{|l|}{ Length of Stay (months) } \\
\hline Mean & 6.1 \\
\hline $\mathrm{SD}$ & 9.0 \\
\hline Range & $1-106$ \\
\hline \multicolumn{2}{|l|}{ Race } \\
\hline African American & $12 \%$ \\
\hline Asian/Pacific & $1 \%$ \\
\hline Caucasian & $75 \%$ \\
\hline Hispanic & $10 \%$ \\
\hline Multi-racial & $1 \%$ \\
\hline Middle Eastern & $1 \%$ \\
\hline \multicolumn{2}{|l|}{ Education } \\
\hline Graduate Degree & $4 \%$ \\
\hline Bachelor's Degree & $15 \%$ \\
\hline AA Degree & $9 \%$ \\
\hline Vocational Tech Degree & $1 \%$ \\
\hline Some College & $7 \%$ \\
\hline High School Diploma & $54 \%$ \\
\hline $9^{\text {th }}-11^{\text {th }}$ Grade & $9 \%$ \\
\hline$<9^{\text {th }}$ Grade & $1 \%$ \\
\hline \multicolumn{2}{|l|}{ Severity of TBI (MPAI-4 rating) } \\
\hline Mild $(<40)$ & $3.0 \%$ \\
\hline Mild-moderate (40 - 49) & $18.0 \%$ \\
\hline Moderate (50 - 59) & $41.0 \%$ \\
\hline Severe $(60+)$ & $38.0 \%$ \\
\hline
\end{tabular}




\subsection{Measure}

Participant functioning was assessed using the Mayo-Portland Adaptability Inventory-4 (MPAI-4) [8] at the time of admission and time of discharge from the treatment facilities involved in the study. Specifically, the MPAI-4 consists of 29 items rated from 0 to 4 on a 5-point scale, where 0 represents no limitations and 4 represents a severe problem interfering with activity more than $75 \%$ of the time. Raw scores on the 29 items are converted to T-scores within three subscales: Ability Index, Adjustment Index, and Participation Index. Each index has an average impairment $\mathrm{T}$-score of 50 and a standard deviation of 10 points. The T-score interpretation is inverted so that higher scores reflect greater disability. The MPAI-4 and its three subscales (Ability, Adjustment, and Participation Indices) offer well developed and documented psychometric properties. The MPAI-4 has undergone rigorous psychometric testing and has proven reliability and validity as determined through Rasch analysis, Item Cluster, Principle Component Analyses (PCA), and measures of concurrent and predictive validity [9] [10].

\subsection{Procedure}

Participants were evaluated upon admission by each program's multidisciplinary treatment team comprised of physicians, nursing, physical therapy, occupational therapy, speech therapy, counseling/psychology, case management, cognitive rehabilitation specialists, and facility-based life skills teachers. Once individual discipline assessments were completed, each participant was then evaluated using the MPAI-4 within 30 days of admission by treatment team consensus. Discharge MPAI-4s were completed in a similar fashion within the final week of the participant's stay. The results of the evaluations were then compiled into a national database.

\subsection{Analysis}

Rasch analysis was performed to determine reliability of MPAI-4 admission and discharge assessments and the goodness of fit for dependent variables used in regression analyses.

\subsection{Construct Validity}

Construct validity refers to the extent to which an evaluation tool measures the underlying construct that it is intended to measure. Rasch fit statistics accomplish this by evaluating expected values for an item to the actual value obtain from the data set. Fit statistics also provide an estimate of the distinct contribution for each item in describing the underlying construct and the extent to which they differentiate among people along the continuum of that construct [10]. As applied to the MPAI-4, Rasch Infit and Outfit statistics illustrate the fit of each item representing unique contribution to a person's level of disability (latent construct). Fit values that are nearest to 1.0 indicate minimal distortion. Values 
falling below 1 indicate that persons are answering incorrectly when they are expected to answer correctly (Guttman error). Low fit values on the MPAI-4 suggest that high levels of limitation are observed when low levels would be expected for that person on those items. Values greater than 1 indicate that there is more noise or random variation on an item than would be expected. Fit values falling between 0.5 and 1.5 are considered productive for measurement use [11]. Items that fall outside those parameters may not reliably represent the latent construct being measured.

\subsection{Reliability}

Reliability refers to the consistency of a measure or the extent to which a measure produces similar results from one testing occasion to another. Key statistics provided by Rasch analysis to evaluate measurement consistency are Person and Item Reliability and Person and Item Separation. Specifically, Person Reliability indicates how well items comprising a measure distinguish among individuals (e.g. those possessing a lot or a little of the construct measured) while Item Reliability refers to whether test items relate to each other in a consistent way in describing a disparate group of individuals. A coefficient of .80 or greater is considered acceptable for Person Reliability, while a coefficient of at least .90 is optimal for Item Reliability [12].

Separation values reveal how well items distinguish among people along a performance continuum (Person Separation) and the unique contribution of items to the construct being measured. Person Separation values indicate the number of performance levels detected by a measure. For example, a Person Separation index of 2.00 means that two levels of performance can be reliably identified.

Item Separation refers to the extent to which items on a test are consistently ranked from least difficult to most difficult. Low Item Separation $(<3.00)$ implies that the item difficulty hierarchy is not reliable, whereas magnitudes exceeding 3.00 indicate greater consistency of item hierarchy.

\subsection{Prediction}

Multiple regression analyses were conducted to identify a predictive model for outcomes following post-hospital residential brain injury rehabilitation. The variables of interest included physical and cognitive functions measured by the MPAI-4 Abilities Index and three person variables: age, length of stay in program, and chronicity (onset of injury to admission).

\subsection{Dependent Variable}

In this study, functional outcome was measured using the following three ordinal variables from the MPAI-4 participation index: initiation, self-care, and residence (home and community skills). These variables were selected because they are fundamental to independent functioning: the ability to react appropriately to 
stimuli in the environment (initiation), the ability to manage personal hygiene (self-care), and the ability to function in the home and community safely and efficiently (residence). Rasch goodness-of-fit statistics were examined for the three variables. Fit statistics falling between 0.50 and 1.5 demonstrate linearity of data, an important assumption for dependent variables used in linear regression models [13], p. 1359). Fit statistics for the three dependent variables were 0.71, 0.87, and 0.73 respectively for initiation, self-care and residence.

Prior to use in linear multiple regression, raw scores for the three ordinal dependent variables were converted to $z$-scores and summed across the three variables to create a single measure of functional outcome. To further establish the linearity of this new variable, $\mathrm{z}$-scores were converted to T-scores yielding a linear dependent variable appropriate use in linear multiple regression [10].

\subsection{Independent Variables}

Variables on the Abilities Index, which provide measures of frontal lobe functioning, were entered as predictor variables in the multiple regression analyses. Each of these variables measure frontal lobe functions that are at the highest level of cognitive processing requiring interaction of multiple systems to produce physical, behavioral, and communication functioning to meet daily challenges [14] [15]. Table 2 lists these variables along with a brief description of each.

Table 2. MPAI-4 abilities items entered into step-wise multiple regression analysis.

\begin{tabular}{|c|c|}
\hline MPAI-4 Abilities Items & Description \\
\hline Mobility & $\begin{array}{l}\text { Problems walking or moving; balance problems that } \\
\text { interfere with moving about }\end{array}$ \\
\hline Use of hands & Impaired strength or coordination one or both hands \\
\hline Vision & $\begin{array}{l}\text { Problems seeing; double vision; eye, brain, or nerve } \\
\text { injuries that interfere with seeing }\end{array}$ \\
\hline Audition & Problems hearing; ringing in the ears \\
\hline Dizziness & Feeling unsteady, dizzy, light-headed \\
\hline Motor speech & Abnormal clearness or rate of speech; stuttering \\
\hline Verbal communication & Problems expressing or understanding language \\
\hline Nonverbal communication & $\begin{array}{l}\text { Restricted or unusual gestures or facial expression; talking } \\
\text { too much or not enough; missing nonverbal cues from others }\end{array}$ \\
\hline Attention/ concentration & $\begin{array}{l}\text { Problems ignoring distractions, shifting attention, } \\
\text { keeping more than one thing in mind at a time }\end{array}$ \\
\hline Memory & Problems learning and recalling new information \\
\hline Fund of Information & $\begin{array}{l}\text { Problems remembering information learned in school or } \\
\text { on the job; difficulty remembering information about } \\
\text { self and family from years ago }\end{array}$ \\
\hline Novel problem solving & $\begin{array}{l}\text { Problems thinking up solutions or picking the best } \\
\text { solution to new problems }\end{array}$ \\
\hline Visuospatial abilities & $\begin{array}{l}\text { Problems drawing, assembling things, route-finding, being } \\
\text { visually aware on both the left and the right sides }\end{array}$ \\
\hline
\end{tabular}

From Lezak \& Malek, 2008. 
In addition to the Abilities index items, the predictive power of age (participant age at admission), length of stay (days in program) and chronicity (onset of injury to admission measured in days) were also examined. Each of these variables has been shown to effect success in rehabilitation [6] [16] [17].

\subsection{Multiple Regression Analyses}

Stepwise multiple regression was the primary method used to identify the best set of predictor variables from those listed in Table 2. The stepwise multiple regression analysis searches for predictive relationships within a data set but is prone to overestimation of the statistical significance between variables yielding results that may be sample dependent (Type 1 error). To reduce this risk, the data set was randomly divided into two independent sub-groups using SPSS version-25. The predictor variables were entered into the equation for the first independent sub-group. To validate the results of the stepwise regression, the significant predictor variables were then entered into a Hierarchical Regression using the second independent sub-group. For further review of procedures for controlling for Type 1 error using multiple regression, the reader is referred to Cohen and Cohen [18].

\section{Results}

\subsection{Rasch Analysis}

Table 3 presents Rasch infit and outfit statistics on MPAI- 4 admission and discharge evaluations for those variables that fell outside the $1.0 \pm 0.5$ parameter established for acceptable fit.

Values for each of these items exceeded 1.5, revealing significant unexplained variation in observations and a tendency for outlier responding. None of the predictor or dependent variables used in the study fell outside of acceptable fit parameters.

\subsection{Reliability}

Rasch person reliability coefficients were 0.90 , and 0.94 respectively for admission and discharge assessments. These reliability coefficients exceeded the acceptable standard of 0.80 for person reliability and indicated the MPAI- 4 effectively discriminated among those along the disability continuum.

Table 3. MPAI-4 items with infit and outfit values outside acceptable parameters.

\begin{tabular}{ccccc}
\hline \multirow{2}{*}{ MPAI-4 Items } & \multicolumn{2}{c}{ Admission } & \multicolumn{2}{c}{ Discharge } \\
\cline { 2 - 5 } & Infit & Outfit & Infit & Outfit \\
\hline Paid work & 2.11 & 1.99 & 1.87 & 1.96 \\
Unpaid work & 1.70 & 2.27 & 1.76 & 1.96 \\
Audition & - & 1.77 & 1.80 & 2.23 \\
\hline
\end{tabular}

-indicates value within acceptable level. 
Item reliability coefficients were 0.99 at each assessment period. These are considered strong reliability coefficients. Therefore, the results suggested that for both assessment periods the items across the disability continuum were stable. There was a high level of agreement in identifying easy through difficult items.

\subsection{Separation}

Rasch person separation values were 2.94 (admission) and 3.81 (discharge). These values indicate that the MPAI- 4 revealed three performance strata (e.g. low, medium, and high disability) at both admission and discharge. The item separation values for both assessment periods exceeded 3.00 indicating a consistent item hierarchy from least difficult to most difficult. Specific item separation values were 22.90 (admission) and 25.31 (discharge). Based on the results, there was sufficient item and person separation for purposes of the analysis.

\subsection{Prediction}

A step-wise multiple regression conducted on 891 of the total sample $(n=1717)$ identified a predictive model with five functional variables (mobility, novel problem solving, non-verbal communication, fund of information, and use of hands) and one demographic variable (chronicity), $\mathrm{R}^{2}=0.45, \mathrm{~F}(6,885)=137.95$, $\mathrm{p}<0.0001$ (adjusted $\mathrm{R}^{2}=0.44$ ). Of those variables, Mobility predicted the largest portion of variance in functional outcome (adjusted $R^{2}=0.26$ ). Novel problem solving at admission increased the prediction $14 \%$. The remaining three functional variables contributed an additional $4 \%$ to the prediction of outcome. Chronicity with a significant beta weight of $0.08(\mathrm{p}<0.01)$ added less than $1 \%$ to the model. The validation hierarchical regression conducted on a second subsample $(n=831)$ with those six variables entered was also significant $\left(R^{2}=0.45\right.$, $\mathrm{F}(6,826)=106.46, \mathrm{p}<0.0001$, adjusted $\left.\mathrm{R}^{2}=0.44\right)$. Again mobility and novel problem solving at admission were the strongest predictors. Age and length of stay were not significant in either of the regression analyses. Table 4 displays the predictive findings for the analyses.

Table 4. Prediction of functional outcome at discharge.

\begin{tabular}{cccccccr}
\hline & \multicolumn{3}{c}{ Stepwise Regression } & \multicolumn{2}{c}{ Hierarchical Regression } \\
\cline { 2 - 7 } Predictors & $\begin{array}{c}\text { R-Square } \\
\text { Added }\end{array}$ & $\begin{array}{c}\text { Cumulative } \\
\text { R-Square }\end{array}$ & $\begin{array}{c}\text { Final } \\
\text { Beta }\end{array}$ & $\begin{array}{c}\text { R-Square } \\
\text { Added }\end{array}$ & $\begin{array}{c}\text { Cumulative } \\
\text { R-Square }\end{array}$ & $\begin{array}{c}\text { Final } \\
\text { Beta }\end{array}$ \\
Mobility & $0.261^{* *}$ & 0.261 & $0.326^{* *}$ & $0.272^{* *}$ & 0.272 & $0.337^{* *}$ \\
Novel Problem Solving & $0.142^{* *}$ & 0.403 & $0.230^{* *}$ & $.125^{* *}$ & 0.397 & $0.178^{* *}$ \\
Fund of Information & $0.010^{* *}$ & 0.413 & $0.120^{* *}$ & $0.024^{* *}$ & 0.421 & $0.175^{* *}$ \\
Use of Hands & $0.010^{* *}$ & 0.423 & $0.114^{* *}$ & $0.010^{* *}$ & 0.431 & $0.129^{* *}$ \\
Nonverbal Communication & $0.020^{* *}$ & 0.443 & $0.139^{* *}$ & $0.010^{*}$ & 0.441 & $0.103^{*}$ \\
Chronicity & $0.006^{*}$ & 0.449 & $0.08^{* *}$ & 0.001 & 0.442 & $0.100^{*}$ \\
\hline
\end{tabular}

${ }^{*} \mathrm{p}<0.01 ;{ }^{* *} \mathrm{p}<0.001$ 


\section{Discussion}

For the majority of patients with acquired brain injury, rehabilitative treatment occurs within a hospital system [7]. Unfortunately many leave the hospital system with chronic disabilities requiring supervision and physical assistance [4] [19]. Over the past 30 years a system of post-hospital rehabilitative care, both residential and nonresidential, has emerged. Substantial research shows the efficacy of this level of care for continuing the reduction of disability that begins in the hospital system [3] [20] [21] [22]. However, limited funding for this specialized care creates the need for evidenced based rehabilitation to further improve treatment efficiency and effectiveness [1]. Targeted treatment may lead to cost effective care with improved access in order to reduce disability.

As in all areas of health care, improving treatment efficacy requires an understanding of disability through the use of accurate, reliable and comprehensive assessment tools. The MPAI-4 meets these requirements. The MPAI-4 assesses 29 areas of disability and has undergone extensive psychometric testing [9]. In the present study, the MPAI-4 admission and discharge assessments exceeded Rasch standards for reliability and validity.

Improving clinical practice also requires identification of variables that have the greatest influence on functional outcome. More importantly research findings need to help clinicians focus efforts directly into skills that have the greatest impact on variables that change a person's level of function and need for assistance. In the present study, functional outcome was determined at discharge by a T-score comprised of three MPAI-4 Participation items: Initiation (the ability to initiate and inhibit responses effectively), Self-Care (the ability to manage personal hygiene), and Residence (the ability to function in the home and community safely and efficiently). Previous research by Lewis and Horn [5] identified these as important determinants of societal participation following brain injury. To better understand how to effect these important variables, the study focused on physical and cognitive contributions from the MPAI-4 Abilities Index. These variables were entered into the multiple regression analyses for prediction of outcome. In two separate regression analyses, the following 5 MPAI-4 Abilities items were revealed to be most influential for positive outcomes related to societal participation: Mobility, Novel Problem Solving, Fund of Information, Use of Hands, and Nonverbal Communication. The two strongest predictors were Mobility (moving about safely and effectively) accounting for $26 \%$ of outcome, and Novel Problem Solving (thinking of and executing solutions to new problems) contributing another $14 \%$ toward outcome. Both are functions of the prefrontal and frontal cortex. The prefrontal cortex controls cognitive processing including which movements may be executed in a given situation. The frontal cortex evaluates internal and external cues to monitor and predict the consequences of an action (e.g., novel problem solving). These combined executive functions are critical for independent living and are reflected in the MPAI-4. Thus the finding that these two items accounted for $40 \%$ of overall outcome is 
not surprising. The findings provide a foundation for being prescriptive with post-hospital rehabilitation, namely, targeting deficit areas that impact disability reduction regardless of injury severity.

\section{Conclusion}

Meaningful best practice employs the translation from retrospective to prospective controlled studies that replicate the current findings and identify optimal treatment frequency and duration based on this model. The results of this study establish a basis for prospective controlled research that can lead to a prescriptive approach to post-hospital brain injury rehabilitation. The goal with the prescriptive approach is evidence-based targeted care with improved outcomes and cost management for greater accessibility for those injured. Two separate regression analyses revealed that $40 \%$ of rehabilitative outcome was attributed to Mobility and Novel Problem Solving. Targeting these functions with additional treatment frequency and duration would likely result in an increase in patient independence at discharge.

\section{Conflicts of Interest}

The authors declare no conflict of interest regarding the publication of this paper.

\section{References}

[1] Masel, B.E. and DeWitt, D.S. (2010) Traumatic Brain Injury: A Disease Process, Not an Event. Journal of Neurotrauma, 27, 1529-1540.

https://www.ncbi.nlm.nih.gov/pubmed/20504161

[2] Hayden, M.E., Plenger, P. and Bison, K.L. (2013) Treatment Effect versus Pretreatment Recovery in Persons with Acquired Brain Injury: A Study Regarding the Effectiveness of Post-Acute Rehabilitation. American Academy of Physical Medicine and Rehabilitation, 5, 319-327. https://doi.org/10.1016/j.pmrj.2012.12.005

[3] Altman, I.M., Swick, S., Parrot, D. and Malec, J.F. (2010) Effectiveness of Community-Based Rehabilitation after Traumatic Brain Injury for 489 Program Completers Compared with Those Precipitously Discharged. Archives of Physical Medicine and Rehabilitation, 91, 1697-1704. https://doi.org/10.1016/j.apmr.2010.08.001

[4] Langlosis, J.A., Rutland-Brown, W. and Wald, M. (2006) The Epidemiology and Impact of Acquired Brain Injury: A Brief Overview. The Journal of Head Trauma Rehabilitation, 21, 375-378. https://doi.org/10.1097/00001199-200609000-00001

[5] Lewis, F. and Horn, G. (2015) Neurologic Continuum of Care: Evidence-Based Model of a Post-Hospital System of Care. Neurorehabilitation, 36, 243-251. https://doi.org/10.3233/NRE-151213

[6] Lewis, F., Horn, G. and Russell, R. (2017) Impact of Chronicity on Outcomes Following Post-Hospital Residential Brain Injury Rehabilitation: Application of Multivariate Statistics and Rasch Analysis. Open Journal of Statistics, 7, 254-263. https://doi.org/10.4236/ojs.2017.72020

[7] Horn, G.J. and Lewis, F.D. (2014) A Model of Care for Neurological Rehabilitation. Journal of Nurse Life Care Planning, 14, 681-692.

[8] Malec, J.F. and Lezak, M.D. (2008) The Mayo-Portland Adaptability Inventory 
(MPAI-4) for Adults. Child Adolescence Manual, 1-84.

http://www.tbims.org/combi/mpai

https://doi.org/10.1037/t29117-000

[9] Kean, J., Malec, J., Altman, I. and Swick, S. (2011) Rasch Measurement Analysis of the Mayo-Portland Adaptability Inventory (MPAI-4) in a Community-Based Rehabilitation Sample. Journal of Neurotrauma, 28, 745-753. https://doi.org/10.1089/neu.2010.1573

[10] Malec, J., Kragness, M., Evans, R., Finlay, K., Kent, A. and Lezak, M. (2003) Further Psychometric Evaluation and Revision of the Mayo-Portland Adaptability Inventory in a National Sample. The Journal of Head Trauma Rehabilitation, 18, 479-492. https://doi.org/10.1097/00001199-200311000-00002

[11] Linacre, J. (2002) What Do Infit, Outfit, Mean-Square, and Standardization Mean? Archives of Rasch Measurement, 16, 871-882.

[12] Andrich, D. (1978) A Rating Formulation for Ordered Response Categories. Psychometrika, 43, 561-573. https://doi.org/10.1007/BF02293814

[13] Tennant, A. and Conaghan, P.G. (2007) The Rasch Measurement Model in Rheumatology: What Is It and Why Use It? When Should It Be Applied and What Should One Look for in a Rasch Paper? Arthritis Care \& Research, 57, 1358-1362. https://doi.org/10.1002/art.23108

[14] Godefroy, O. (2003) Frontal Syndrome and Disorders of Executive Functions. Journal of Neurology, 250, 1-6. https://doi.org/10.1007/s00415-003-0918-2

[15] McDonald, B.C., Flashman, L.A. and Saykin, A.J. (2002) Executive Dysfunction Following Traumatic Brain Injury: Neural Substrates and Treatment Strategies. NeuroRehabilitation, 17, 333-344.

[16] Hawkins, M.L., Lewis, F.D. and Medeiros, R. (1996) Serious Traumatic Brain Injury: An Evaluation of Functional Outcomes. The Journal of Trauma: Injury and Critical Care, 41, 257-283. https://doi.org/10.1097/00005373-199608000-00010

[17] Reeder, K., Rosenthal, M., Lichtenberg, P. and Wood, D. (1996) Impact of Age on Functional Outcome Following Traumatic Brain Injury. Journal of Head Trauma Rehabilitation, 11, 22-31. https://doi.org/10.1097/00001199-199606000-00006

[18] Cohen, J. and Cohen, P. (1975) Applied Multiple Regression/Correlation Analysis for the Behavioural Sciences. Lawrence Erlbaum, Hillsdale.

[19] Cope, N.D., Cole, J.R., Hall, K.M. and Barkans, H. (1991) Brain Injury: Analysis of Outcome in a Post-Acute Rehabilitation System. Part I: General Analysis. Brain Injury, 5, 111-125. https://doi.org/10.3109/02699059109008083

[20] Horn, G.J., Lewis, F.D., Russell, R. and Kemp, D. (2017) Anxiety Following Traumatic Brain Injury: Impact on Post-Hospital Rehabilitation Outcomes. Physical Medicine \& Rehabilitation Research, 2, 1-6. https://doi.org/10.15761/PMRR.1000149

[21] Lewis, F. and Horn, G. (2017) Rasch Analysis and Functional Measurement in Post-Hospital Brain Injury Rehabilitation. International Journal of Statistics and Probability, 6, 50-59. https://doi.org/10.5539/ijsp.v6n6p50

[22] Malec, J.E. and Brasford, J.S. (1996) Post-Acute Brain Injury Rehabilitation. Archives of Physical Medicine and Rehabilitation, 77, 198-207.

https://doi.org/10.1016/S0003-9993(96)90168-9 